\title{
A high-Nb TiAl alloy with highly refined microstructure and excellent mechanical properties fabricated by electromagnetic continuous casting
}

\author{
Yong-zhe Wang, *Hong-sheng Ding, Rui-run Chen, Jing-jie Guo, Heng-zhi Fu, and Jin-peng Lü \\ National Key Laboratory for Precision Hot Processing of Metals, School of Materials Science and Engineering, Harbin Institute of Technology, \\ Harbin 150001, China
}

\begin{abstract}
In the present research, microstructure refinement of a high-Nb TiAl alloy (Ti-48Al-8Nb-0.15B ) was realized by means of the electromagnetic continuous casting (EMCC) technique. The microstructure of an ingot obtained by EMCC was analyzed using scanning electron microscopy (SEM). As compared with the raw as-cast alloy, the obtained EMCC alloy presented a much finer microstructure with lamellar colonies with a mean size of about $50-70 \mu \mathrm{m}$ because the electromagnetic stirring broke initial dendrites and enhanced the heterogeneous nucleation. As the grains were refined, the properties of the TiAl alloy were improved significantly. This implies that the EMCC technique could offer the possibility of application for high-Nb TiAl alloys with a refined microstructure and excellent properties to be used as a structural material.
\end{abstract}

Key words: high-Nb TiAl alloy; microstructure; electromagnetic continuous casting; mechanical properties

CLC numbers: TG146.23 Document code: A Article ID: 1672-6421 2016 05-342-04

$\mathrm{H}$ igh-Nb-TiAl alloys have been widely investigated due to their excellent strength, creep resistance and oxidation resistance at elevated temperatures ${ }^{[1]}$. Therefore, the development of such a material system has important implications for aeronautics and astronautics fields. However, poor ductility at room temperature as well as low workability at elevated temperatures of the high-Nb TiAl alloys restricts their applications ${ }^{[2]}$. According to current knowledge, high mechanical properties can be achieved in these materials by attaining a finer fully lamellar microstructure ${ }^{[3]}$.

Electromagnetic continuous casting (EMCC) is a technique which is in virtue of electromagnetic force to strengthen the movement of the metallic melt and improve heat transfer during solidification. When the EMCC is applied to the melt during solidification, the

\footnotetext{
*Hong-sheng Ding

Male, born in 1968, Ph.D, Professor. His main research fields include solidification and microstructure control, investment casting technology of titanium-aluminum alloys and directionally solidified titanium-aluminum alloys. E-mail: dinghsh@hit.edu.cn

Corresponding author: Yong-zhe Wang

E-mail address: hitwangyz@163.com
}

Received: 2015-10-22; Accepted: 2016-04-23 microstructure would be refined and the distribution of compositions also could be homogenized ${ }^{[4-6]}$. EMCC technique has been applied to the production of many alloys such as aluminum alloys ${ }^{[4]}$, magnesium alloys ${ }^{[5]}$ and alloyed steels ${ }^{[6]}$, but application to TiAl alloys has rarely been reported.

Based on the above opinions, the EMCC technique was used in refining a high-Nb TiAl alloy with the composition of Ti-48Al-8Nb-0.15B in the present study, and the microstructure and mechanical properties of EMCCed samples were investigated.

\section{Experimental procedure}

A high-Nb TiAl intermetallic alloy with a chemical composition of Ti-48Al-8Nb-0.15B was melted using pure metals in a cold crucible induction furnace and cast into a steel mold. The obtained ingot, $140 \mathrm{~mm}$ in diameter and $300 \mathrm{~mm}$ in length, was cut along its axis into rods about $20 \mathrm{~mm}$ in diameter and $250 \mathrm{~mm}$ in length using a wire electrical discharge machine. Subsequently, these rods were remelted using a set of electromagnetic continuous casting equipment. The EMCC process is schematically shown in Fig. 1 and details about the working procedure can be found in a previous study by Z. Z. Shen ${ }^{[2]}$. 
The experiment equipment of the EMCC process used in the present study, and schematically shown in Fig. 1, consists of a square crystallizer with an inner dimensions of $26 \mathrm{~mm} \times 26 \mathrm{~mm}$ and a four-turn induction coil arranged outside the crystallizer. The coil has access to high-frequency alternating current with a frequency of was $50 \mathrm{kHz}$. Throughout the process of the experiment, the coil current intensity was maintained at 5,000 A, and the melting pool was induction heated by an electromagnetic field that was generated by the alternating current. The upper rod for feeding and the base for solidifying simultaneously moved down at $2.1 \mathrm{~mm} \cdot \mathrm{min}^{-1}$ and $1.0 \mathrm{~mm} \cdot \mathrm{min}^{-1}$, respectively. Liquid Ga-In was used to cool the hot ingot at the bottom.

The microstructures of raw as-cast and EMCC ingots were observed using a field emission scanning electron microscope (SEM) in the back scattered electron imaging (BSE) mode. Transmission electron microscopy (TEM) analysis was performed to determine the crystal structure of boride precipitates. Compression tests on samples in raw as-cast and EMCC high-Nb TiAl alloys with a dimension of $\Phi 3 \mathrm{~mm} \times 4.5$ $\mathrm{mm}$ at an initial strain rate of $1 \times 10^{-3} \mathrm{~s}^{-1}$ were carried out using an Instron 5569 testing machine.

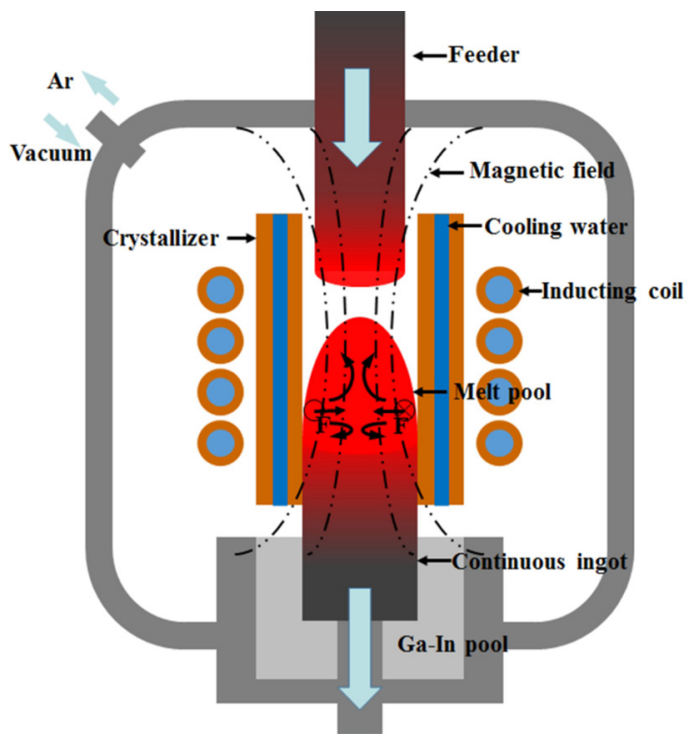

Fig. 1: Schematic of electromagnetic continuous casting process

\section{Results and discussion}

The macrostructures of the raw as-cast Ti-48Al-8Nb-0.15B ingot before EMCC were observed at different locations, as shown in Fig. 2. At the rim of the ingot, the macrostructure consists of large columnar grains oriented inwards and parallel to the direction of heat flow (Fig. 2a). Similar observations were also made for ternary and multicomponent alloys solidifying via primary $\alpha$ phase, which have been reported in studies for raw ascast specimens ranging from arc-melted buttons to large ingots ${ }^{[7,8]}$. Figure 2(b) shows equiaxed grains at the center of this ingot. Shrinkage and pores were also observed and indicated by arrows. These casting defects were formed as a result of insufficient feeding during the solidification process, and have significant influence on the room temperature properties.
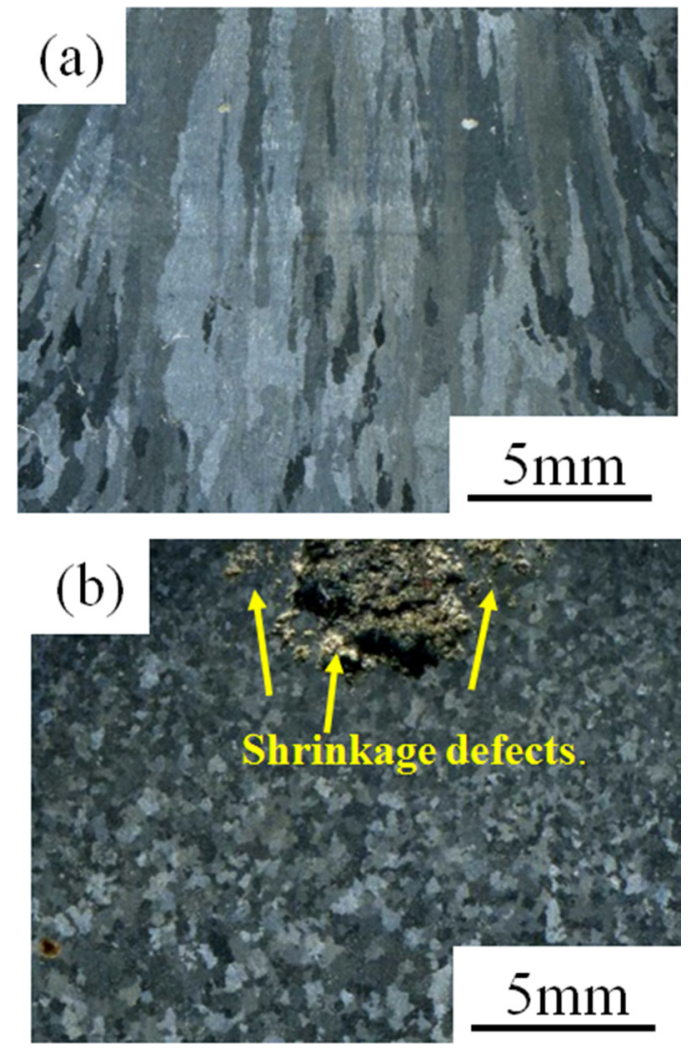

Fig. 2: Macrostructures of raw as-cast Ti-48Al-8Nb-0.15B alloy at rim (a) and center (b)

Figure 3(a) shows the EMCC Ti-48Al-8Nb-0.15B alloy ingot. It can be seen that the surface of the ingot is smooth and has no obvious surface flaws and crack defects. Figure 3(b) shows the macrostructure in the longitudinal section of the continuous casting zone as marked in Fig. 3(a). Compared with raw ascast alloy, the EMCC ingot consists of a large number of fine equiaxed grains with a compact structure and without defects. These results show that the EMCC technique can ensure the progressive solidification of the casting, eliminate shrinkage and porosity, and generate obvious grain refining effect. As shown in Fig. 3(c), the EMCC ingot has a microstructure with much finer $\alpha_{2} / \gamma$ lamellar colonies of about $50-70 \mu \mathrm{m}$. It can also be seen from this figure that some shorter needle-like borides are distributed in or through lamellar colonies and less than $50 \mu \mathrm{m}$ in length. Figure 3(d) shows the TEM micrograph of boride and its corresponding electron diffraction pattern. These precipitates have been identified as titanium monoboride with a B27 structure. Figure 3(e) is the macrostructure in the longitudinal section of the "1" zone indicated in Fig. 3(a), which is obtained by suddenly cutting off the current. Figures 3 (f) and (g) are the high magnification micrographs of the " 2 " and "3" zones indicated in Fig. 3(e), respectively. Figure 3(f) is the microstructure after current interruption. In this figure, $\alpha$-dendrites with the secondary dendrite arms approximately $60^{\circ}$ to the primary arms were observed. In addition, the microstructure composed of equiaxed hexagonal $\alpha$ phase in Fig. $3(\mathrm{~g})$ indicates that the $\alpha$-dendrites were broken when EMCC was applied. 

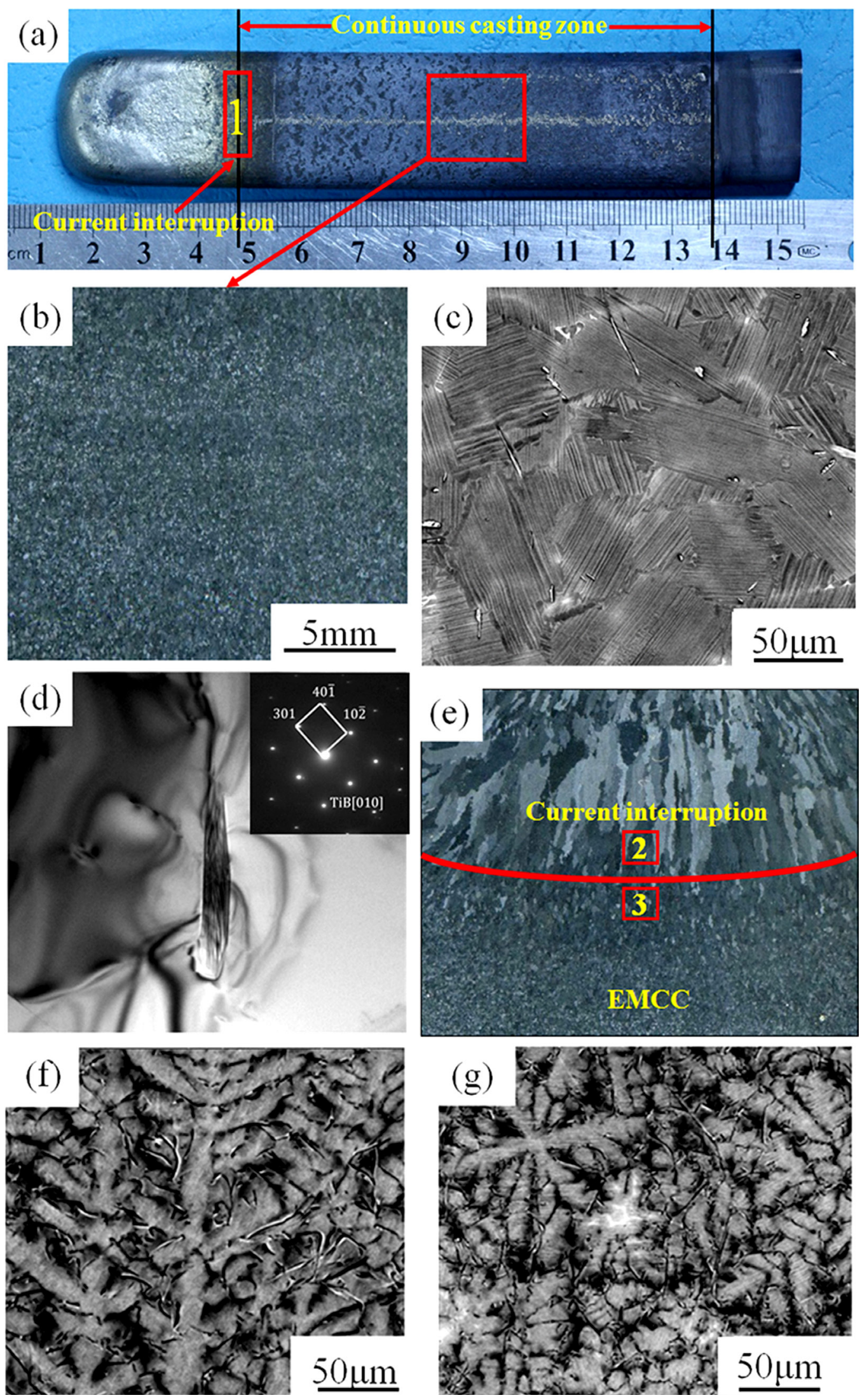

Fig. 3: Structure of EMCCed Ti-48Al-8Nb-0.15B alloy: (a) continuous casting ingot; (b) macrostructure; (c) microstructure of continuous casting zone; (d) TEM micrograph of boride and its corresponding electron diffraction pattern; (e) macrostructure of "1" zone indicated in Fig. 3(a); (f) and (g) SEM micrographs of "2" and " 3 " zones indicated in Fig. $3(e)$, respectively

In general, boron is added to TiAl alloys to act as a grainrefining alloying element. However, the grain refinement cannot be observed in Fig. 2, indicating that adding only $0.15 \%$ boron is not sufficient to refine the structure of Ti-48Al-8Nb alloy. 
Chen also found that less than 0.3 at $\%$ boron could not refine the grain size in Ti-44Al-8Nb alloy ${ }^{[9]}$. In the present study, adding trace boron aims to obtain dendrite arms with weakened bases, which could be easily broken by electromagnetic stirring in the EMCC process, and the broken-off fragments of dendrite could be responsible for grain refinement.

In the EMCC process, alternative magnetic field was generated by the alternative current. An electromagnetic force $F$ owing to the induced magnetic field was produced in the melt and affected the dendrite growth. The force consists of two parts expressed as follows ${ }^{[10]}$ :

$$
F=-\nabla B^{2} / 2 \mu+(B \nabla) B / \mu
$$

where $B$ is the induced magnetic flux density, and $\mu$ is the magnetic permeability of TiAl melt. The first part of this equation is a potential force to sustain the melt. This force is beneficial for building up the surface pressure to form softcontact and improve the surface quality. The second part is a rotational component which results in a forced convection in the melt. This force stirs the melt and refines the structure. The refinement mechanism of EMCC has been extensively studied ${ }^{[4-6,10]}$. This effect is mainly attributed to the brokenoff fragments of dendrites, which is caused by the forced convection during dendritic growth, and subsequently the detached branches are carried away by convection and dispersed in the molten pool, therefore promoting the heterogeneous nucleation. Meanwhile, the uniform temperature distribution caused by the action of electromagnetic stirring is also beneficial in suppressing the growth of dendrites and the formation of solidification cracks. The above factors contribute to grain refinement and reduction of casting defects.

Figure 4 shows the typical compressive stress-strain curves of the raw as-cast and the EMCC high-Nb TiAl alloy samples. As compared with the raw as-cast sample, the EMCC TiAl alloy sample has higher yield strength (703 MPa), compressive strength $(2,200 \mathrm{MPa})$ and fracture strain $(34.77 \%)$. It can be concluded that EMCC technique can improve the mechanical

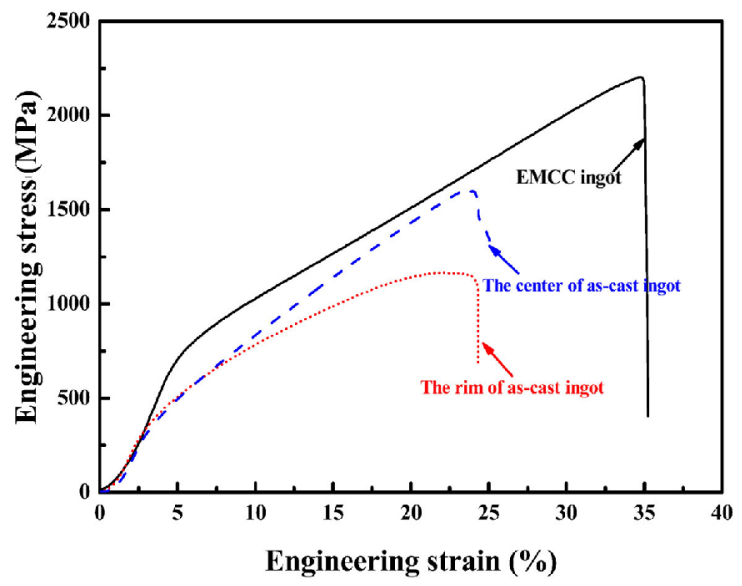

Fig. 4: Typical compressive stress-strain curves of raw ascast and EMCCed high-Nb TiAl alloy samples properties of Ti-48Al-8Nb-0.15B alloy. Strengthening mainly arises from the decrease in lamellar colony size and fine boride particles, which are impenetrable for dislocations ${ }^{[11,12]}$.

\section{Conclusions}

(1) The EMCC ingot's microstructure is characterized by fine $\alpha_{2} / \gamma$ lamellar colonies and limited borides. The lamellar colonies are homogeneous and have a dimension of about 50-70 $\mu \mathrm{m}$, and the length of borides is less than $50 \mu \mathrm{m}$.

(2) The refinement mechanism is mainly attributed to the action of electromagnetic stirring during the EMCC process, which breaks $\alpha$-dendrites and promotes heterogeneous nucleation.

(3) Compared with raw as-cast alloy samples, the mechanical performance of the EMCC high-Nb TiAl alloy samples have been improved owing to the refined microstructure.

\section{References}

[1] Zhao Ertuan, Kong Fantao, Chen Yanfei, et al. Characterization of zirconia-based slurries with different binders for titanium investment casting. China Foundry, 2012, 9(2): 125-130.

[2] Shen Z Z, Lin J P, Liang Y F, et al. A novel hot pack rolling of high $\mathrm{Nb}-\mathrm{TiAl}$ sheet from cast ingot. Intermetallics, 2015, 67: 19-25.

[3] Wu XH and Hu D. Microstructural refinement in cast TiAl alloys by solid state transformations. Scripta Materialia, 2005, 52(8): 731-734.

[4] Zhang J, Kang S B, Yu H S, et al. Effect of fine-grained raw material addition on microstructure refinement and tensile properties in horizontal continuous casting $\mathrm{Al}-12 \% \mathrm{Si}$ alloy billets. Materials \& Design, 2011, 32(6): 3566-3569.

[5] Kwak T Y, Kim W J. Mater. Hot compression behavior of the 1 wt $\%$ calcium containing Mg-8Al-0.5Zn (AZ80) alloy fabricated using electromagnetic casting technology. Materials Science and Engineering A, 2014, 615(6): 222-230.

[6] Li J C, Wang B F, Ma Y L, et al. Effect of complex electromagnetic stirring on inner quality of high carbon steel bloom. Materials Science and Engineering A, 2006, 425(1-2): 201-204.

[7] Imayev R M, Imayev V M, Oehring $M$, et al. Alloy design concepts for refined gamma titanium aluminide based alloys. Intermetallics, 2007, 15(4): 451-460.

[8] Zollinger J, Lapin J, Daloz, D, et al. Influence of oxygen on solidification behaviour of cast TiAl-based alloys. Intermetallics, 2007, 15(10): 1343-1350.

[9] Chen T T. The mechanism of grain refinement in TiAl alloys by boron addition - an alternative hypothesis. Intermetallics, 2000, 8(1): 29-37.

[10] Yu J B, Jiang J M, Ren Z M, et al. A new method of continuous casting of copper billets by a combination of $A C$ current and magnetic fields. Materials \& Design, 2009, 30(10): 4565-4569.

[11] Wu $Y$ and Hwang S K. Microstructural refinement and improvement of mechanical properties and oxidation resistance in EPM TiAl-based intermetallics with yttrium addition. Acta Materialia, 2002, 50(6): 1479-93.

[12] Hu D, Mei J F, Wichins M, et al. Microstructure and tensile properties of investment cast Ti-46Al-8Nb-1B alloy. Scripta Materialia, 2002, 47(4): 273-278. 